\title{
Discovery of $\beta$ Cep pulsations in the eclipsing binary V453 Cygni
}

\author{
John Southworth ${ }^{\oplus}{ }^{1 \star}$ D. M. Bowman, ${ }^{2}$ A. Tkachenko ${ }^{2}$ and K. Pavlovski ${ }^{3}$ \\ ${ }^{1}$ Astrophysics Group, Keele University, Staffordshire ST5 5BG, UK \\ ${ }^{2}$ Institute of Astronomy, KU Leuven, Celestijnenlaan 200D, B-3001 Leuven, Belgium \\ ${ }^{3}$ Department of Physics, Faculty of Science, University of Zagreb, Bijenicka cesta 32, 10000 Zagreb, Croatia
}

Accepted 2020 May 14. Received 2020 May 11; in original form 2020 April 8

\begin{abstract}
V453 Cyg is an eclipsing binary containing 14 and $11 \mathrm{M}_{\odot}$ stars in an eccentric short-period orbit. We have discovered $\beta$ Cep-type pulsations in this system using Transiting Exoplanet Survey Satellite data. We identify seven significant pulsation frequencies, between 2.37 and $10.51 \mathrm{~d}^{-1}$, in the primary star. These include six frequencies that are separated by yet significantly offset from harmonics of the orbital frequency, indicating they are tidally perturbed modes. We have determined the physical properties of the system to high precision: V453 Cyg A is the first $\beta$ Cep pulsator with a precise mass measurement. The system is a vital tracer of the physical processes that govern the evolution of massive single and binary stars.
\end{abstract}

Key words: binaries: eclipsing-stars: fundamental parameters - stars: oscillations.

\section{INTRODUCTION}

Massive stars - those with initial masses $\gtrsim 8 \mathrm{M}_{\odot}$ - are important drivers in the chemical and dynamical evolution of galaxies (Langer 2012). Despite their importance in the cosmos, we still lack prescriptions for their interior rotation, mixing, and angular momentum transport mechanisms (Aerts, Mathis \& Rogers 2019; Aerts 2020). Since massive stars harbour convective cores during the hydrogencore burning phase of stellar evolution, mixing in the near-core region directly supplies fresh hydrogen to the core and prolongs the main-sequence lifetime. However, the amount of near-core mixing within massive stars is observationally unconstrained, which makes it challenging to accurately and reliably infer the absolute ages of single stars from only their location in the HertzsprungRussell (HR) diagram. The physical correlations and degeneracies between model parameters in current stellar evolution codes are more important for massive stars in binary systems since many of these will interact during their lifetimes (Sana et al. 2012).

One of the best methods for breaking model degeneracies is by using eclipsing binary stars (EBs), as their masses and radii can be measured entirely empirically and to high precision, and because we can assume that the stars have the same age and chemical composition (Torres, Andersen \& Giménez 2010). The physical properties can be compared to the predictions of theoretical evolutionary models to infer the age and composition of the components, and to investigate the effectiveness of different parametrizations of the interior physics implemented in the codes. However, there is often significant disagreement between the masses of $\mathrm{O}$ and B stars inferred from spectroscopy and stellar evolution theory, versus dynamical masses measurements in binary systems. This

^E-mail: astro.js@keele.ac.uk mass discrepancy was identified by Herrero et al. (1992) and has been explained by invoking varying amounts of near-core mixing (Guinan et al. 2000; Tkachenko et al. 2014, 2016, 2020).

Among massive stars are a type of pulsating variables known as $\beta$ Cep stars, which have masses of $8-15 \mathrm{M}_{\odot}$ (Aerts, ChristensenDalsgaard \& Kurtz 2010). These stars pulsate in low radial order gravity ( $\mathrm{g}$ ) and pressure (p) modes with pulsation periods of several hours and pulsation amplitudes up to a few tenths of a magnitude (Stankov \& Handler 2005). Pulsations have great potential in providing constraints on stellar interiors, since each pulsation mode is sensitive to a specific pulsation cavity within a star. For example, the identification of rotationally split $\mathrm{p}$ modes has allowed the interior rotation to be determined (Aerts et al. 2003; Briquet et al. 2007) and the impact of magnetic fields to be constrained (Shibahashi \& Aerts 2000; Briquet et al. 2013). The launch of space missions such as $C o R o T$, Kepler, and $K 2$ has allowed many new pulsating massive stars to be discovered (e.g. McNamara, Jackiewicz \& McKeever 2012; Neiner et al. 2012; Pápics et al. 2017; Burssens et al. 2019).

A unique opportunity exists in the study of pulsating stars in binary systems. Here, the measured masses and radii fix the positions of the stars in the mass-radius and HR diagrams, while the pulsations allow a direct probe of interior rotation and mixing. Furthermore, pulsating binary systems have the potential to constrain the effect of tides on stellar structure and evolution by means of tidally induced pulsations in eccentric systems (Welsh et al. 2011; Hambleton et al. 2013; Beck et al. 2014; Fuller 2017; Handler et al. 2020), and the recently discovered tidally perturbed pulsations in short-period binaries (Bowman et al. 2019a). However, no precise mass measurement currently exists for a $\beta$ Cep pulsator. A few have been found in binary systems, for example EN Lac (Jerzykiewicz et al. 2015), V381 Car (Freyhammer et al. 2005) and $\alpha$ Vir (Tkachenko et al. 2016), but in each case the mass is not precisely known because either the secondary star is not detectable 
in spectra or the orbital inclination is poorly determined due to the lack of eclipses.

In this work, we present the discovery of $\beta$ Cep pulsations in V453 Cyg, an EB consisting of B0.4 IV + B0.7 IV stars. Its orbit is slightly eccentric, has a period of $3.89 \mathrm{~d}$, and exhibits apsidal motion with a short apsidal period (Wachmann 1973). It is a member of NGC 6871, a sparse open cluster that is the nucleus of Cyg OB3 (Hoag et al. 1961; Garmany \& Stencel 1992). V453 Cyg was discovered to be eclipsing by Wachmann (1939), and extensive photometry of the system has been published by Wachmann (1974), Cohen (1974), and Zakirov (1992). Spectroscopic orbits have been obtained by Popper \& Hill (1991), Simon \& Sturm (1994), and Burkholder, Massey \& Morrell (1997), chemical abundances by Pavlovski \& Southworth (2009) and Pavlovski, Southworth \& Tamajo (2018), and physical properties by Southworth, Maxted \& Smalley (2004) and Pavlovski et al. (2018).

\section{OBSERVATIONS}

V453 Cyg was observed using the NASA Transiting Exoplanet Survey Satellite (TESS; Ricker et al. 2015). TESS is observing the majority of the sky, with each hemisphere divided into 13 sectors based on ecliptic longitude. Observations in each sector last for $27 \mathrm{~d}$, with an interruption for data download near the midpoint.

V453 Cyg was observed in Sectors 14 and 15, between 2019 July 18 and September 11, at a cadence of 2 min. Fig. 1 shows the SAP (simple aperture photometry) and PDC (pre-search data conditioning) light curves (Jenkins et al. 2016).

We applied the least-squares deconvolution technique (Tkachenko et al. 2013) to two published sets of spectra (Southworth et al. 2004; Pavlovski et al. 2018) and find clear signs of line profile variations (LPVs) in the primary star. We therefore attribute the pulsations to this star, but caution that the data were not good enough to demonstrate that the secondary star is not pulsating.

\section{PHYSICAL PROPERTIES OF V453 CYG}

\subsection{Preliminary light-curve analysis: JKTEBOP}

The TESS light curves of V453 Cyg were initially modelled using the JKTEBOP code $^{1}$ (Southworth 2008, 2013). This is a fast code that provides a good fit to the eclipses, so is useful for normalizing the data to zero differential magnitude and for isolating the pulsation signatures. However, it approximates the stars as spheres, so the parameters of the fit are not reliable for stars as distorted as those in V453 Cyg.

We fitted both the PDC and SAP light curves, but our final results rest on the SAP data as the systematic effects are smaller in this light curve; use of the PDC data has no significant effect on our conclusions. Due to the sometimes large systematics, we divided the SAP data into chunks of contiguous data points and included a polynomial function of second or third order to fit the out-of-eclipse brightness in each. There are 22473 SAP data points in our fits.

We fitted for the sum and ratio of the fractional radii of the stars $\left(r_{\mathrm{A}}=\frac{R_{\mathrm{A}}}{a}\right.$ and $r_{\mathrm{B}}=\frac{R_{\mathrm{B}}}{a}$, where $R_{\mathrm{A}}$ and $R_{\mathrm{B}}$ are the true radii and $a$ is the orbital semimajor axis), the orbital inclination, the central surface brightness ratio, orbital period, and a reference time of mid-transit. We did not use historical light curves or times

${ }^{1}$ http: //www.astro.keele.ac.uk/jkt/codes/jktebop.ht $\mathrm{ml}$ of minimum light in order to avoid the complexities induced by the apsidal motion of the system. Limb darkening was included using the quadratic law with the linear coefficients fitted and the quadratic coefficients fixed to values from Claret (2017). The orbital eccentricity $(e)$ and argument of periastron $(\omega)$ were fitted using the Poincaré elements $(e \cos \omega$ and $e \sin \omega)$.

In order to account for the pulsations simultaneously with the effects of binarity, we added to JKTEBOP the option to fit for one or more sine terms applied to various parameters. We experimented with including one sine term applied to the light of the system or of either of the two stars. All three options yielded a significantly better fit to the data, but are statistically indistinguishable, so could not be used to confirm the spectroscopic result that the primary star is the pulsator in the system.

\subsection{Final light-curve analysis: Wilson-Devinney}

The TESS light curve was fitted using the Wilson-Devinney (WD) code (Wilson \& Devinney 1971), which uses Roche geometry to accurately model the observable properties of binary systems. We used the 2004 version of this code, driven via the JKTWD wrapper (Southworth et al. 2011). Due to the much longer computing time needed for the WD code, particularly in the case of an eccentric system, we modelled a light curve that had already been cleaned using JKTEBOP. This cleaning process comprised normalization to zero differential magnitude, removal of the sinusoidal variation due to the strongest pulsation, and phase-binning into 300 data points.

This binned light curve was modelled with JKTWD using the same approach as adopted in Pavlovski et al. (2018), with the one modification that we always fitted for the light output of the two stars separately because the TESS passband is not implemented in the 2004 version of the WD code. The control and fixed parameters of the final fit are given in Table 1; see Wilson \& Van Hamme (2004) for a detailed description of each quantity. The Poisson noise in the light curve is negligible, so the uncertainties in the parameters derived from the data are dominated by choices made during the modelling process. In order to capture these uncertainties, we ran solutions with different approaches to the treatment of eccentricity, third light, rotation rate, albedo, gravity darkening, treatment of limb darkening, and treatment of reflection. The adopted model and the uncertainties from the variety of models considered are collected in Table 1. We found it important to include albedo as a fitted parameter, as this significantly decreased the residuals of the fit. The albedo values are positively correlated with the assumed gravity darkening exponents. The best fit is shown in Fig. 2, where the residuals come primarily from incomplete removal of the pulsations at this stage.

\subsection{Physical properties of V453 Cyg}

The light-curve parameters found in the previous section were augmented by the velocity amplitudes $\left(K_{\mathrm{A}}=175.2 \pm 1.3 \mathrm{~km} \mathrm{~s}^{-1}\right.$ and $\left.K_{\mathrm{B}}=220.2 \pm 1.6 \mathrm{~km} \mathrm{~s}^{-1}\right)$ and effective temperatures $\left(T_{\text {eff } \mathrm{A}}=\right.$ $28800 \pm 500 \mathrm{~K}$ and $T_{\text {eff B }}=27700 \pm 600 \mathrm{~K}$ ) of the stars. These were taken from Pavlovski et al. (2018), who obtained them by disentangling the spectra (Simon \& Sturm 1994; Ilijić et al. 2004) and fitting them with a grid of non-LTE synthetic spectra. We then used the JKTABSDIM code (Southworth, Maxted \& Smalley 2005) to calculate the physical properties of the system, propagating the uncertainties using a perturbation analysis. The distance to the system was calculated from its BVRI apparent magnitudes, the bolometric corrections from Girardi et al. (2002), and by manual 


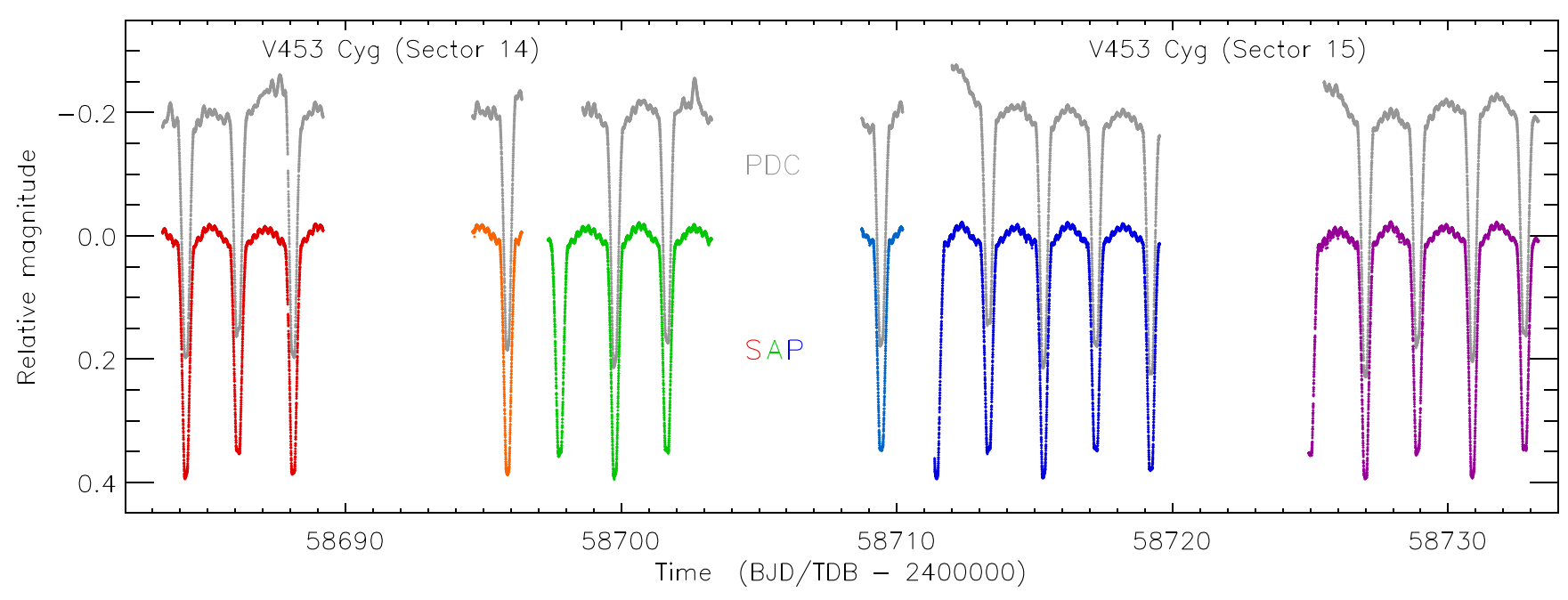

Figure 1. TESS light curve of V453 Cyg. The PDC data are shown in grey and the SAP data are shown in six colours. The colours indicate the division of the data into six sets for the polynomial normalization functions.

Table 1. Brief summary of the parameters for the WD solution of the TESS light curve of V453 Cyg. Uncertainties are only quoted when they have been robustly assessed by comparison between a full set of alternative solutions.

\begin{tabular}{|c|c|c|}
\hline Parameter & Star A & Star B \\
\hline \multicolumn{3}{|l|}{ Control parameters } \\
\hline WD2004 operation mode & \multicolumn{2}{|c|}{0} \\
\hline Treatment of reflection & \multicolumn{2}{|c|}{1} \\
\hline Number of reflections & \multicolumn{2}{|c|}{1} \\
\hline Limb darkening law & \multicolumn{2}{|c|}{1 (linear) } \\
\hline $\begin{array}{l}\text { Numerical grid size } \\
\text { (normal) }\end{array}$ & \multicolumn{2}{|c|}{60} \\
\hline Numerical grid size (coarse) & \multicolumn{2}{|c|}{40} \\
\hline \multicolumn{3}{|l|}{ Fixed parameters } \\
\hline Mass ratio & \multicolumn{2}{|c|}{0.795} \\
\hline Rotation rates & 1.0 & 1.4 \\
\hline Gravity darkening & 1.0 & 1.0 \\
\hline$T_{\text {eff }}(\mathrm{K})$ & 28800 & 27700 \\
\hline \multicolumn{3}{|l|}{ Fitted parameters } \\
\hline Orbital inclination $\left(^{\circ}\right)$ & \multicolumn{2}{|c|}{$86.14 \pm 0.30$} \\
\hline Orbital eccentricity & \multicolumn{2}{|c|}{$0.0250 \pm 0.0014$} \\
\hline Argument of periastron $\left({ }^{\circ}\right)$ & \multicolumn{2}{|c|}{$152.5 \pm 5.1$} \\
\hline Third light & \multicolumn{2}{|c|}{$0.002 \pm 0.013$} \\
\hline Light contributions & $9.079 \pm 0.020$ & $3.026 \pm 0.035$ \\
\hline Potential & $4.383 \pm 0.013$ & $5.762 \pm 0.043$ \\
\hline Bolometric albedos & $1.33 \pm 0.44$ & $1.07 \pm 0.35$ \\
\hline Fractional radii & $0.2844 \pm 0.0010$ & $0.1723 \pm 0.0016$ \\
\hline \multicolumn{3}{|l|}{ Derived parameters } \\
\hline Orbital separation $\left(\mathrm{R}_{\odot}\right)$ & \multicolumn{2}{|c|}{$30.47 \pm 0.16$} \\
\hline $\operatorname{Mass}\left(\mathrm{M}_{\odot}\right)$ & $13.96 \pm 0.23$ & $11.10 \pm 0.18$ \\
\hline Radius $\left(\mathrm{R}_{\odot}\right)$ & $8.665 \pm 0.055$ & $5.250 \pm 0.056$ \\
\hline Log surface gravity (cgs) & $3.708 \pm 0.004$ & $4.044 \pm 0.009$ \\
\hline$T_{\text {eff }}(\mathrm{K})$ & $28800 \pm 500$ & $27700 \pm 600$ \\
\hline Log luminosity $\left(\mathrm{L}_{\odot}\right)$ & $4.666 \pm 0.031$ & $4.163 \pm 0.039$ \\
\hline $\begin{array}{l}\text { Absolute bolometric } \\
\text { magnitude }\end{array}$ & $-6.914 \pm 0.077$ & $-5.657 \pm 0.097$ \\
\hline Distance (pc) & \multicolumn{2}{|c|}{$1851 \pm 60$} \\
\hline
\end{tabular}

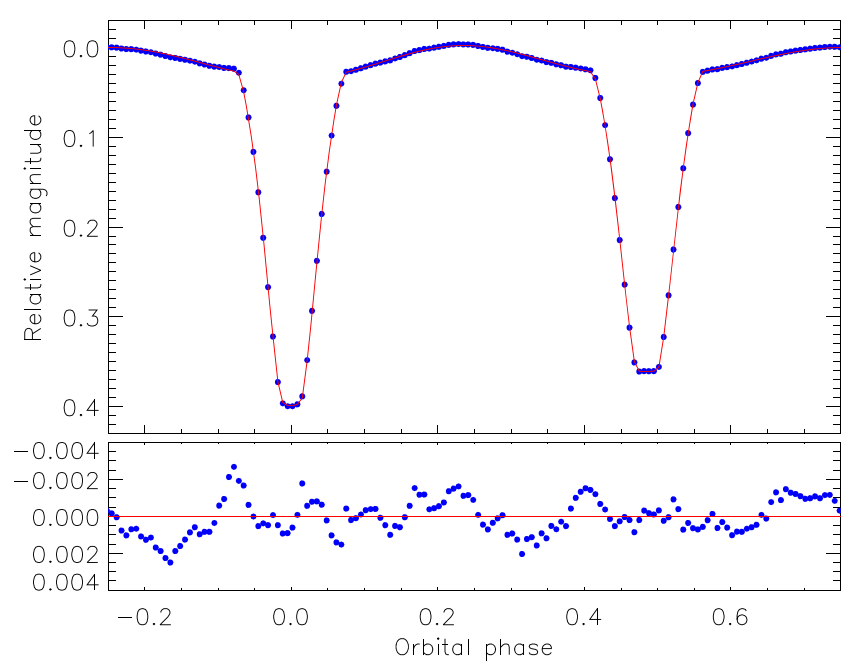

Figure 2. Phase-binned SAP light curve of V453 Cyg (filled blue circles) with the WD2004 best fit (red solid line). The residuals of the fit are shown on a large scale in the lower panel.

iteration of the reddening parameter $E_{B-V}=0.44 \pm 0.03$, to bring the distances from different passbands into agreement.

The masses of the stars are measured to an accuracy of 1.6 per cent and their radii to 0.6 and 1.0 percent. The JKTEBOP code gives radii that differ from those found using WD2004 by 2 per cent. We interpret this as an indication that JKTEBOP does not give reliable parameters for stars as tidally distorted as those of V453 Cyg. The two codes agree much better for stars with smaller fractional radii, e.g. to within 0.1 percent when $r_{\mathrm{A}}=0.04$ and $r_{\mathrm{B}}=0.06$ (Maxted et al. 2020).

The pseudo-synchronous rotational velocities of the primary and secondary stars are $112.7 \pm 0.7$ and $68.3 \pm 0.7 \mathrm{~km} \mathrm{~s}^{-1}$, respectively, whereas the $v \sin i$ values measured by Pavlovski et al. (2018) are $107.2 \pm 2.8$ and $98.3 \pm 3.7 \mathrm{~km} \mathrm{~s}^{-1}$. We therefore agree with the conclusion of Pavlovski et al. that the primary star is rotating approximately pseudo-synchronously with the orbit, whereas the secondary star is rotating faster than this by a factor of $1.44 \pm 0.08$. 
Table 2. Frequencies, amplitudes, and phases of significant frequencies in $\mathrm{V} 453 \mathrm{Cyg}$. The $1 \sigma$ uncertainties from the multifrequency non-linear leastsquares fit are given in parentheses. The amplitude, $\mathrm{S} / \mathrm{N}$, and the difference between each frequency and a harmonic, $i$, of the orbital frequency are given.

\begin{tabular}{lccrcr}
\hline $\begin{array}{l}\text { Frequency } \\
\left(\mathrm{d}^{-1}\right)\end{array}$ & $\begin{array}{c}\text { Amplitude } \\
(\mathrm{mmag})\end{array}$ & $\begin{array}{c}\text { Phase } \\
(\mathrm{rad})\end{array}$ & $\mathrm{S} / \mathrm{N}$ & $i$ & \multicolumn{1}{c}{$\begin{array}{c}v-i v_{\text {orb }} \\
\left(\mathrm{d}^{-1}\right)\end{array}$} \\
\hline $1.02900(18)$ & $0.983(17)$ & $-1.32(3)$ & 5.2 & 4 & $0.0007(8)$ \\
$1.54215(23)$ & $0.766(17)$ & $-1.20(4)$ & 5.0 & 6 & $-0.0003(8)$ \\
$2.37468(42)$ & $0.423(17)$ & $1.63(8)$ & 4.0 & 9 & $0.0610(9)$ \\
$2.88971(35)$ & $0.504(17)$ & $-1.11(7)$ & 4.6 & 11 & $0.0618(9)$ \\
$3.66118(63)$ & $0.285(17)$ & $1.55(12)$ & 4.0 & 14 & $0.0621(10)$ \\
$4.17402(49)$ & $0.366(17)$ & $1.93(9)$ & 4.1 & 16 & $0.0607(10)$ \\
$4.43103(19)$ & $0.924(17)$ & $0.23(4)$ & 10.5 & 17 & $0.0607(8)$ \\
$4.94605(6)$ & $3.099(17)$ & $0.52(1)$ & 30.4 & 19 & $0.0615(8)$ \\
$10.50733(68)$ & $0.262(17)$ & $-1.55(13)$ & 6.8 & 41 & $-0.0330(11)$ \\
\hline & & & & &
\end{tabular}

\section{PULSATION ANALYSIS}

After subtracting the binary model from the TESS light curve (Section 3.1), we used a discrete Fourier transform (Deeming 1975; Kurtz 1985) to calculate the amplitude spectrum of the remaining variability in V453 Cyg. The 2-min cadence TESS data have a Nyquist frequency of $360 \mathrm{~d}^{-1}$. We extracted all significant pulsation modes, defined as having an amplitude signal-to-noise ratio $(\mathrm{S} / \mathrm{N})$ larger than 4 (Breger 1993), using iterative pre-whitening, and then performed a multifrequency non-linear least-squares fit to optimize their parameters (Kurtz et al. 2015; Bowman 2017). The nine significant frequencies are given in Table 2.

Two of the frequencies in Table 2, 1.03 and $1.54 \mathrm{~d}^{-1}$, are within $3.7 \sigma$ and $1.5 \sigma$ of the fourth and sixth harmonics of the orbital frequency, respectively. These frequencies could be tidally induced modes, but, given the extremely high precision of the TESS light curve, we cannot exclude that they result from the subtraction of an imperfect binary model, which leaves residual signal at orbital harmonics. The highest frequency, $10.51 \mathrm{~d}^{-1}$, is not a combination of any other frequency and likely an independent heat-driven $\mathrm{p}$ mode. Assuming the $1 \sigma$ confidence intervals in the masses and radii from our binary model, we estimate using pulsation constants and the period-mean density relation (see e.g. Bowman 2017) that the fundamental p-mode frequency is 4.07(7) and 7.7(2) $\mathrm{d}^{-1}$ in the primary and secondary, respectively. Thus, the extracted frequencies represent low radial order $g$ and $\mathrm{p}$ modes.
The TESS data alone do not allow us to firmly ascertain which star is pulsating. We do not find amplitude modulation of the pulsation modes during the orbit, so V453 Cyg is not a single-sided pulsator (Handler et al. 2020). However, we do find regularity among six significant frequencies, which exhibit a quasi-constant offset from orbital frequency harmonics and are indicative of tidally perturbed pulsations (Bowman et al. 2019a). The amplitude spectra before and after pre-whitening are shown in the top and bottom panels of Fig. 3, respectively, in which the locations of extracted frequencies are shown as colour-coded vertical lines. The vertical dashed grey lines indicate multiples of the orbital frequency and illustrate the regularity of the tidally perturbed pulsation modes.

As demonstrated by Fig. 3, there is considerable variance remaining in the residual amplitude spectrum of V453 Cyg, but it is not significant given our amplitude $\mathrm{S} / \mathrm{N} \geq 4$ criterion. The regularity among mode frequencies is clearly related to the orbital frequency of the system, with a common spacing of approximately $0.51 \mathrm{~d}^{-1}$ (i.e. twice the orbital frequency, which is related to the rotation frequency in a pseudo-synchronized binary). We also note that the amplitude distribution of the remaining variance is similar to the recent near-ubiquitous detection of gravity waves excited by core convection in hundreds of massive stars (Bowman et al. 2019b). The presence of this stochastic low-frequency variability remains significant up to $\sim 30 \mathrm{~d}^{-1}$ above the white noise level, which we calculate to be approximately $13 \mu$ mag.

\section{SUMMARY AND DISCUSSION}

V453 Cyg is an extensively studied EB containing stars of masses 14 and $11 \mathrm{M}_{\odot}$, in a short-period orbit that shows eccentricity and apsidal motion. Using TESS photometry, we have discovered $\beta$ Cep pulsations in this eclipsing system. LPVs in published spectroscopy indicate that these pulsations arise from the primary star. We have used the TESS light curve and published spectroscopic results to determine the physical properties of the stars to high precision. We subtracted the effects of binarity from the light curve to leave behind the pulsation signal, and then subjected this to a frequency analysis.

We found tidally perturbed pulsations similar to those recently discovered in the Algol system U Gru (Bowman et al. 2019a). Similarly, it is plausible that the tidal torque in V453 Cyg is strong enough to perturb pulsation modes and cause regularity in the amplitude spectrum in the form of pulsation frequencies offset from orbital harmonics separated by the orbital frequency. However,

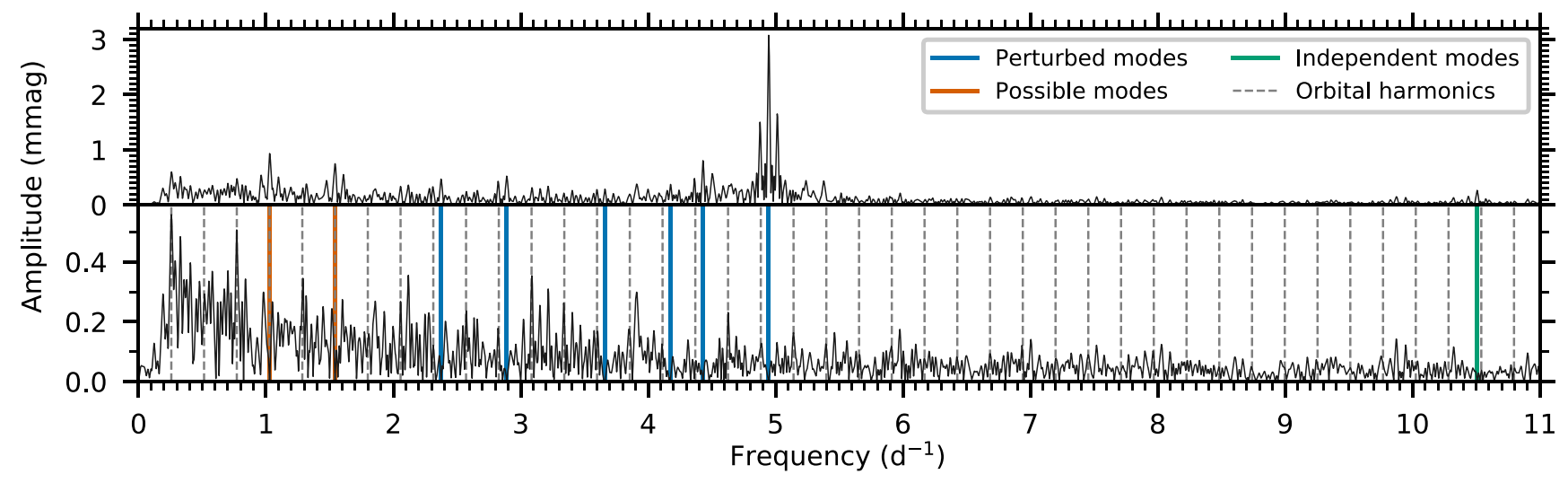

Figure 3. Amplitude spectra of V453 Cyg after removal of the binary model (top), and also the significant pulsation frequencies (bottom). The location and identity of extracted frequencies are shown as coloured vertical lines, and the vertical dashed grey lines represent multiples of the orbital frequency. 
there are important differences between these systems. U Gru is experiencing slow mass transfer, has a circular orbit, and shows $\delta$ Sct pulsations. V453 Cyg has undergone no mass transfer, its eccentric orbit means the tidal deformation of the stars changes continuously through each orbit, and its larger stellar masses mean the tidally perturbed pulsation frequencies are in the $\beta$ Cep frequency regime.

It is important to obtain further spectroscopic data for V453 Cyg to confirm that the primary star is pulsating, to check whether the secondary star is contributing any of the pulsation frequencies, and to measure the component masses to better than the 1 percent precision level necessary for the highest-fidelity tests of theoretical models (e.g. Valle et al. 2018). A new apsidal motion study would also be valuable. Armed with these empirical measurements, V453 Cyg represents a unique opportunity for a detailed theoretical exploration of the physics that governs the structure and evolution of massive stars in short-period binary systems.

\section{ACKNOWLEDGEMENTS}

The TESS data presented in this paper were obtained from the Mikulski Archive for Space Telescopes (MAST) at the Space Telescope Science Institute (STScI). STScI is operated by the Association of Universities for Research in Astronomy, Inc. Support to MAST for these data is provided by the NASA Office of Space Science. Funding for the TESS mission is provided by the NASA Explorer Program. The research leading to these results has received funding from the European Research Council (ERC) under the European Union's Horizon 2020 research and innovation programme (grant agreement no. 670519: MAMSIE), from the KU Leuven Research Council (grant C16/18/005: PARADISE), from the Research Foundation Flanders (FWO) under grant agreement G0H5416N (ERC runner-up project), from the BELgian federal Science Policy Office (BELSPO) through PRODEX grant PLATO.

\section{REFERENCES}

Aerts C., 2020, preprint (arXiv:1912.12300)

Aerts C., Thoul A., Daszyńska J., Scuflaire R., Waelkens C., Dupret M. A., Niemczura E., Noels A., 2003, Science, 300, 1926

Aerts C., Christensen-Dalsgaard J., Kurtz D. W., 2010, Asteroseismology (Astronomy and Astrophysics Library). Springer-Verlag, Berlin

Aerts C., Mathis S., Rogers T. M., 2019, ARA\&A, 57, 35

Beck P. G. et al., 2014, A\&A, 564, A36

Bowman D. M., 2017, Amplitude Modulation of Pulsation Modes in Delta Scuti Stars, Springer Theses Series. Springer-Verlag, Berlin

Bowman D. M., Johnston C., Tkachenko A., Mkrtichian D. E., Gunsriwiwat K., Aerts C., 2019a, ApJ, 883, L26

Bowman D. M. et al., 2019b, Nat. Astron., 3, 760

Breger M., 1993, Ap\&SS, 210, 173

Briquet M., Morel T., Thoul A., Scuflaire R., Miglio A., Montalbán J., Dupret M. A., Aerts C., 2007, MNRAS, 381, 1482

Briquet M., Neiner C., Leroy B., Pápics P. I., MiMeS Collaboration, 2013, A\&A, 557, L16

Burkholder V., Massey P., Morrell N., 1997, ApJ, 490, 328

Burssens S., Bowman D. M., Aerts C., Pedersen M. G., Moravveji E., Buysschaert B., 2019, MNRAS, 489, 1304

Claret A., 2017, A\&A, 600, A30

Cohen H. L., 1974, A\&AS, 15, 181

Deeming T. J., 1975, Ap\&SS, 36, 137

Freyhammer L. M., Hensberge H., Sterken C., Pavlovski K., Smette A., Ilijić S., 2005, A\&A, 429, 631
Fuller J., 2017, MNRAS, 472, 1538

Garmany C. D., Stencel R. E., 1992, A\&AS, 94, 211

Girardi L., Bertelli G., Bressan A., Chiosi C., Groenewegen M. A. T., Marigo P., Salasnich B., Weiss A., 2002, A\&A, 391, 195

Guinan E. F., Ribas I., Fitzpatrick E. L., Giménez Á., Jordi C., McCook G. P., Popper D. M., 2000, ApJ, 544, 409

Hambleton K. M. et al., 2013, MNRAS, 434, 925

Handler G. et al., 2020, Nat. Astron., in press

Herrero A., Kudritzki R. P., Vilchez J. M., Kunze D., Butler K., Haser S., 1992, A\&A, 261, 209

Hoag A. A., Johnson H. L., Iriarte B., Mitchell R. I., Hallam K. L., Sharpless S., 1961, Publ. U.S. Nav. Obs. Second Ser., 17, 344

Ilijić S., Hensberge H., Pavlovski K., Freyhammer L. M., 2004, in Hilditch R. W., Hensberge H., Pavlovski K., eds, ASP Conf. Ser. Vol. 318, Spectroscopically and Spatially Resolving the Components of the Close Binary Stars. Astron. Soc. Pac., San Francisco, p. 111

Jenkins J. M. et al., 2016, in Chiozzi G., Guzman J. C., eds, Proc. SPIE Conf. Ser. Vol. 9913, Software and Cyberinfrastructure for Astronomy IV. SPIE, Bellingham, p. 99133E

Jerzykiewicz M. et al., 2015, MNRAS, 454, 724

Kurtz D. W., 1985, MNRAS, 213, 773

Kurtz D. W., Shibahashi H., Murphy S. J., Bedding T. R., Bowman D. M., 2015, MNRAS, 450, 3015

Langer N., 2012, ARA\&A, 50, 107

McNamara B. J., Jackiewicz J., McKeever J., 2012, AJ, 143, 101

Maxted P. F. L. et al., 2020, preprint (arXiv:2003.09295)

Neiner C., Mathis S., Saio H., Lovekin C., Eggenberger P., Lee U., 2012, A\&A, 539, A90

Pápics P. I. et al., 2017, A\&A, 598, A74

Pavlovski K., Southworth J., 2009, MNRAS, 394, 1519

Pavlovski K., Southworth J., Tamajo E., 2018, MNRAS, 481, 3129

Popper D. M., Hill G., 1991, AJ, 101, 600

Ricker G. R. et al., 2015, J. Astron. Telesc. Instrum. Syst., 1, 014003

Sana H. et al., 2012, Science, 337, 444

Shibahashi H., Aerts C., 2000, ApJ, 531, L143

Simon K. P., Sturm E., 1994, A\&A, 281, 286

Southworth J., 2008, MNRAS, 386, 1644

Southworth J., 2013, A\&A, 557, A119

Southworth J., Maxted P. F. L., Smalley B., 2004, MNRAS, 351, 1277

Southworth J., Maxted P. F. L., Smalley B., 2005, A\&A, 429, 645

Southworth J. et al., 2011, MNRAS, 414, 2413

Stankov A., Handler G., 2005, ApJS, 158, 193

Tkachenko A., Van Reeth T., Tsymbal V., Aerts C., Kochukhov O., Debosscher J., 2013, A\&A, 560, A37

Tkachenko A. et al., 2014, MNRAS, 438, 3093

Tkachenko A. et al., 2016, MNRAS, 458, 1964

Tkachenko A. et al., 2020, A\&A, 637, A60

Torres G., Andersen J., Giménez A., 2010, A\&AR, 18, 67

Valle G., Dell'Omodarme M., Prada Moroni P. G., Degl'Innocenti S., 2018, A\&A, 615, A62

Wachmann A. A., 1939, Beob. Zirk. Astron. Nachr., 21, 36

Wachmann A. A., 1973, A\&A, 25, 157

Wachmann A. A., 1974, A\&A, 34, 317

Welsh W. F. et al., 2011, ApJS, 197, 4

Wilson R. E., Devinney E. J., 1971, ApJ, 166, 605

Wilson R. E., Van Hamme W., 2004, Computing Binary Star Observables (Wilson-Devinney Program User Guide). Available at: ftp://ftp.astro.uf 1.edu/pub/wilson

Zakirov M. M., 1992, Kinematika Fiz. Nebesnykh Tel, 8, 38

This paper has been typeset from a $\mathrm{T}_{\mathrm{E}} \mathrm{X} / \mathrm{L} \mathrm{T} \mathrm{E} \mathrm{X}$ file prepared by the author. 